\title{
Data models, visual representations, metaphors: how to solve the puzzle?
}

\author{
T. Catarci出, M.F. Costabile*, I.F. Cruz", Y. Ioannidis ${ }^{\star}$, and B. Shneiderman ${ }^{\star}$ \\ ¿Dipartimento di Informatica e Sistemistica, Università degli Studi di Roma \\ "La Sapienza"
}

Via Salaria, 113 - 00198 Roma, Italy,e-mail: catarci@infokit.dis.uniroma1.it

* Dipartimento di Informatica, Università di Bari

Via Orabona 4, 70126 Bari, Italy, e-mail: fcosta@ercole.ba.cnr.it

- Computer Sciences Departmen, University of Wisconsin

1210 W. Dayton St., Madison, WI 53706, USA, e-mail: yannis@cs.wisc.edu

"Department of Electrical Engineering and Computer Science, Tufts University Medford, 02155 MA, USA, e-mail: isabel@cs.tufts.edu

${ }^{\star}$ Department of Computer Science, University of Maryland

College Park, MD 20742,USA, e-mail: ben@cs.umd.edu

\begin{abstract}
The need of a precise definition of the concepts of metaphor and visual representation in the database realm was widely discussed in the VDB3 Panel. Also, the issue of singling out the relationships existing between the above two concepts and the data model was addressed. An introduction by the panel organizer together with a short position statement by each panelist are reported in the following document.
\end{abstract}

Data model, metaphor, visual representation.

Keywords

\section{INTRODUCTION}

The need of a better interaction between users and database systems has been widely recognized and discussed. It is generally accepted that the quality of the interaction mainly depends on the interface characteristics. Thus, the crucial point is to develop good interfaces, where the word "good" is to be interpreted from the user's perspective. Now, the point is to understand which parts of this interaction belong to the interface and which to the database system.

A database is typically defined in terms of a data model, but the abstract concepts of such a model need to be expressed through a representation in order to be perceived by a user. Any representation of a model aims at stressing some basic features of the model itself, and obviously a many-to-many correspondence exists between data models and representations (visual or not). The representation should be part of the interface, while the data model should be part of the database system. On the other hand, there are data models, the so called semantic models, which 
convey more information to the user by offering richer mechanisms in order to structure the data contained in the database and to show more relationships among them. Also, a representation can be effective, but cannot add semantics to the data. Finally, in addition to data models and representations, a well known component of any interface to any system is the metaphor. Unfortunately, the experience in the other computer science areas does not help us very much. Indeed, most of the famous interface metaphors have been introduced for interaction purposes, i.e., in order to explain some new kind of task through well known actions performed in familiar environment. A typical example is the desktop metaphor. On the contrary, the metaphors we need in developing interfaces to database systems have to deal firstly with the problem of representing the database content, then with the interaction mechanisms.

As a consequence of the above, the basis for the design and construction of database interfaces is still largely ad hoc. We do not yet know:

- how data models, representations, and metaphors interact;

- how to model metaphors and (visual) representations formally,

- how to design metaphors and representations with confidence in both their usability and effectiveness and their adequacy to the underlying data models.

- how to construct systems to incorporate metaphors and representations efficiently and with adequate modularity to support modification and reuse.

Nevertheless, we hope this panel will give some hints on these open questions.

\section{EXPLOITING METAPHORS IN DATABASE INTERFACES (by Maria}

\section{F. Costabile)}

Many authors agree that the metaphor is a tool for creative interface design that makes information easy to find and use, but little research on systematic use of metaphor in interface design exists. This is also true for database interfaces, even if examples of use of metaphors in this field already exist in the literature. Often, metaphors are exploited to help representing the intensional part of the database, i.e. the data schema; in such cases, the metaphor mediates between the data model and the user. However, the user might have a certain mental model of the reality of interest, which is not necessarily reflected by the metaphor used for showing the data model.

A different choice relies on avoiding the visualization of any data model. One possibility is to offer to the user a scenario where the information contained in the database is pictorially represented as a virtual reality, so that the user is no longer aware of a presence of a structured database, but he or she is interacting as in the real world. In this case there is a metaphor from the designer point of view, to bring out of the database (which is not the reality but an internal representation of it) the reality that was meant to be represented when constructing the database.

The very problem I like to point out is that, in many cases, database designers have exploited metaphors that are easily understandable by scientists, such as the table or the node-and-arc metaphor. Unfortunately, the literal meaning of such metaphors is still too far from most nonexpert users. The power of the metaphor is to understand something (the target concept) in terms of something else (the source concept), therefore it is essential to know the source domain at a certain level of detail in order to benefit from the metaphor. Showing on the screen an E-R diagram (even if powerful interaction mechanisms to focus on some portion of the diagram, to scroll a large diagram, etc. are provided) does not really help many users, who are not familiar at all with such a model. If we want information systems to become as easy to manipulate as automobiles are easy to drive, or washing machine are easy to operate, we have to invent more effective metaphors that are closer to the users. This can be achieved by looking carefully at the users, the task they perform, their habits, their preferences, the things they are familiar with. Once potential metaphors have been identified, they need to be tested with users to check if they 
support the users' understanding of the system. Metaphors originating from a source domain that is not easily understandable by the users must be discarded. Therefore, user observation and testing are key elements for an effective metaphorical design.

\section{TAILORABLE VISUALIZATIONS AND META-QUERYING (by Isabel}

\section{F. Cruz)}

New database applications, such as multimedia and scientific applications generate and use large amounts of textual and quantitative data that need to be comprehended by specialists in the different application domains. One possible way to help the users is to display the data in a visual form. For example, if the data is quantitative, plot charts or bar charts can provide a quick overview of the data. Only rarely do users know in advance exactly what data they want to visualize, or how they want to visualize the data. One key issue is to allow for the user to select and process just the relevant data. Powerful database query languages are therefore needed. Another key issue is related to the visualization of the data. We argue that the user should not be limited to a single visual representation (or even to a pre-specified set of visual representations of the data).

Previous research in visual query languages has focused on pre-defined visual representations of data and queries, which are suitable for specific applications, but difficult to extend and generalize. The queries themselves, although visually expressed, conform to the same predefined visual representations.

We have been investigating a system based on a meta-query language, DOODLE, which supports a variety of powerful visual query languages that are user-defined. The visual query languages we are investigating allow the user to give a high-level visual specification of the relevant subset of the database. Using an interactive approach, the user can then further specify new visual queries, causing the display to change accordingly, and to possibly evolve from one representation (e.g., graph) to another representation (e.g., temporal chart). We are using DOODLE in software engineering and graph drawing applications.

In the context of DOODLE, many issues pertaining to the choice of visual representations arise. We have been investigating the expressiveness of visual representations, e.g., the adequacy of a visual representation to encode a given set of database facts. With DOODLE it is also possible to express transformations between visual representations. This raises the issue whether two visual languages have the same "capacity" to express data. We are laying a formal framework that addresses these questions.

\section{DESK-TOP SCHEMA MANAGERS (by Yannis Ioannidis)}

As database schemas increase in complexity and the user base of database systems widens, a pressing need emerges for the development of improved techniques and tools for schema management, and primarily schema visualization. Although existing database systems and prototypes support graphical user interfaces where schemas are represented visually, most of them only provide a single hard-wired such representation, without flexibility.

What we need are Desk-Top Schema Managers that are external to the database system proper, and consist of generic code - not tied to any particular data model or visualization style. Such a system may be built following a framework that, analogously to database models and schemas, introduces visual models (formal visual representation styles) and visual schemas (actual visualizations, instantiations of the visual model). A visual metaphor can then be defined as a mapping from (the elements of) a visual model to (the elements of) a data model. Through this mapping, one is able to assign meaning to a visualization (visual schema) in terms of the 
underlying data schema. Moreover, both the visual and data models and the visual metaphor may be specified declaratively, so that schema visualization is not realized through code that is hardwired in a system, but is established externally in an extensible way. Thus, one may specify different visual models and corresponding visual metaphors to a data model, making it possible to visualize schemas of a given data model in different ways. Another benefit of the formalism is that one can enhance schema visualizations with user-specific annotations and aesthetic preferences, information that is unrelated to the underlying schema.

Desk-Top Schema Managers built based on the above methodology will be able to read external specifications of data and visual models and metaphors and essentially 'become' managers of schemas in these models interpreted by these metaphors. Users will then be able to create, modify, and explore database schemas using any visual representation they find most appropriate. The behavior of the system will also be determined by the characteristics of the input models and metaphors, in the sense that model/metaphor-specific buttons, menu entries, etc. will only be constructed at run-time.

Opossum is a Desk-Top Schema Manager that is being developed at the Univ. of Wisconsin following the above visualization formalism. It offers the spectrum of functionality mentioned above and is currently being used by scientists in Soil Sciences, Genetics, and Biochemistry to design schemas in various models, using a variety of visual models and metaphors.

\section{A NEW INTERFACE GENERATION (by Ben Shneiderman)}

Our recent projects have spawned a new conception of the future of computing. These new ideas allow designers to couple the remarkable human ability at visual information processing with the increasing capacity of computers to display high resolution dynamic color images, in order to ideate a new generation of graphical and direct manipulation user interfaces.

As a first step in this direction, we have developed Visual Information Seeking principles (VIS) to support fact-finding and browsing by novices and experts. We begin with an overview, and then allow users to filter out unwanted data, zoom in on relevant items, and then get detailson-demand. The interface components are tightly coupled to give users appropriate feedback, facilitate query refinement, and prevent errors. Our contributions include dynamic query methods with continuous visual presentation of results as the query is changed (using sliders, buttons, maps), color-coded 2-D space-filling treemaps to show hierarchies in a single display (hundreds of directories and more than 3000 files can be seen at once), and the starfield display (a zoom-able 2-D display with thousands of selectable points of light).

Our work is also concentrated on the Visual Information Explorer (VIZER), a multi-year project to develop advanced software and user interfaces that will allow novice and expert users to visually explore very large databases, spreadsheets, geographic information, temporal sales data, financial reports, and medical information. VIZER's components include the filter/flow representation of boolean operations, dynamic queries that allow small changes in input variables to be shown immediately by changes in a 2- or 3- dimensional display, tree-maps that allow a space filling representation of trees with 3-5000 nodes on the screen at once, and remote visualization that allows users to browse through telepresence. Our goals include the construction of an integrated tool to support exploration, retrieval, gathering, traversal, and capture with goal-directed task-level actions for saving, printing, and sending text, numeric data, images, sound, and other structured information through highspeed distributed networks.

\section{AUTHORS'BIOGRAPHIES}

\section{Tiziana Catarci}


Tiziana Catarci was graduated in Electrical Engineering from the University of Rome "La Sapienza," Rome, Italy, on 1987. She received the Ph.D. degree in Computer Science from the University of Roma "La Sapienza", 1992.

Since 1987 she teaches courses in Computer Science at Italian universities. From 1987 to 1990 she was research assistant at the University of Roma "La Sapienza", where she is now an assistant professor.

Her main research interest is in visual formalisms for database representation and querying. Moreover, she worked in semantic database modeling, cooperative information systems, statistical databases, methodologies for database design. Dr. Catarci has published over sixty papers on the above topics, and written or edited four books.

She is member of the Association for Computing Machinery (ACM), and the Institute of Electrical and Electronics Engineers (IEEE).

\section{Maria F. Costabile}

Maria Francesca Costabile received her doctoral degree in Mathematics at the Universita' della Calabria in 1977. From 1989 she is associate professor at the Department of Computer Science of the University of Bari, Italy.

From 1978 to 1988 she worked at the Dipartimento di Matematica, Universita' della Calabria, Italy, first as postdoctoral fellow and later as university researcher. She has been visiting scientist in several foreign universities. Her research activity was initially in image analysis and computer vision. Her current interests include visual languages, visual interfaces, visual languages for querying databases, formal methods for human-computer interaction, user models. She has published several papers on the above topics.

She is member of the Association for Computing Machinery (ACM), the Institute of Electrical and Electronics Engineers (IEEE) and the Italian Chapter of the International Association on Pattern Recognition (IAPR).

\section{Isabel F. Cruz}

Isabel F. Cruz is an Assistant Professor in the Department of Electrical Engineering and Computer Science at Tufts University. Previously she was a Postdoc in the Department of Computer Science at Brown University. She received her Ph.D. in Computer Science from the University of Toronto in 1993.

Her research interests include databases, user interfaces, multimedia, graph drawing, visual languages, software visualization, and constraint programming.

Currently she is the co-guest editor of the special issue of the Journal of Visual Languages and Computing on Graph Visualization, the co-chair of the 1995 International Workshop on Constraints for Graphics and Visualization and of the 1995 ACM Workshop on Effective Abstractions in Multimedia, and in the program committees of several international conferences. Her research has been funded by the Government of Canada, NATO, and ARPA.

\section{Yannis Ioannidis}

Yannis Ioannidis received his Diploma degree in electrical engineering from the National Technical University of Athens, Greece, in 1982, the M.Sc. degree in applied mathematics (computer science) from Harvard University in 1983, and the Ph.D. degree in computer science from the University of California at Berkeley in 1986.

Since then, he has been on the faculty of the Computer Sciences Department of the University of Wisconsin at Madison, where he is currently an Associate Professor. 
His research interests include query optimization, scientific databases, intelligent/graphical user interfaces, knowledge bases, and heterogeneous databases. He has written over 35 articles on these topics and is a co-author of a patent that is pending.

Dr. Ioannidis is the recipient of an NSF Presidential Young Investigator (PYI) award for 1991-1996. He is currently an Associate Editor of several journals and has been a member of the program committees of over twenty conferences. Dr. Ioannidis is a member of the IEEE Computer Society, the Association of Computing Machinery, and the Technical Chamber of Greece.

\section{Ben Shneiderman}

Ben Shneiderman is a Professor in the Department of Computer Science, Head of the HumanComputer Interaction Laboratory, and Member of the Institute for Systems Research, all at the University of Maryland at College Park. He has taught previously at the State University of New York and at Indiana University. He regularly teaches popular short courses and organizes an annual satellite television presentation on "User Interface Strategies" since 1987.

Dr. Shneiderman is the author of several books on HCI, in addition he edited three technical books, published more than 170 technical papers and book chapters.

Ben Shneiderman has been on the Editorial Advisory Boards of nine journals including the newly formed ACM Transactions on Computer-Human Interaction and the ACM Interactions. He edits the Ablex Publishing Co. book series on "Human-Computer Interaction." He has consulted and lectured for many organizations including Apple, AT\&T, Citicorp, GE, Honeywell, IBM, Intel, Library of Congress, NASA, NCR, and university research groups. 